\title{
VP2 Gene of a Canine Parvovirus Isolate from Stool of a Puppy
}

\author{
Kentarou HIRAYAMA ${ }^{1}$, Rui KANO ${ }^{1)}$, Tomoko HOSOKAWA-KANAI ${ }^{3)}$, Koutarou TUCHIYA ${ }^{3)}$, Shingo TSUYAMA ${ }^{4)}$, \\ Yuka NAKAMURA ${ }^{2)}$, Yoshihide SASAKI ${ }^{2)}$ and Atsuhiko HASEGAWA ${ }^{1)}$ \\ ${ }^{1)}$ Departments of Pathobiology, ${ }^{2)}$ Internal Medicine, Nihon University School of Veterinary Medicine, 1866, Kameino, Fujisawa, \\ Kanagawa, 252-8510, ${ }^{3}$ Nippon Institute for Biological Science, 9-2221-1, Shin-machi, Ome, Tokyo, 198-0024 and ${ }^{4)}$ Tsuyama Animal \\ Hospital, 1-39-1 Yahata, Chigasaki, Kanagawa, 253-0085, Japan
}

(Received 2 February 2004/Accepted 9 September 2004)

ABSTRACT. VP2 gene of a canine parvovirus (CPV) isolate from the feces of a puppy which was diagnosed to be CPV infection was analysed. The result indicated that this clinical isolate was phylogenetically close to the isolate of wild-type CPV (strain CPV-T37) prevailing in Taiwan rather than isolates from Japan.

KEY WORDS: canine parvovirus, molecular analysis, VP2.

J. Vet. Med. Sci. 67(1): 139-143, 2005

Canine parvovirus type2 (CPV-2) emerged as a new pathogen of dogs in 1978, almost simultaneously in Europe and North America, and was responsible for hemorrhagic gastroenteritis and myocarditis in puppies [14, 15]. The close antigenic and genomic relationships among CPV-2, feline panleukopenia virus and mink enteritis virus suggested that CPV-2 might have appeared by genetic mutation in a wild carnivore host receptive to one of the feline panleukopenia virus-like parvoviruses [7, 18].

From 1979 to 1981 , monoclonal antibodies, restriction enzyme analysis, and DNA sequencing demonstrated the antigenic variant or biotype (CPV-2a) that differs from the original strain CPV-2 in three coding regions of the gene for the VP2 capsid protein. A second biotype (CPV-2b) was detected around 1984, and the significant differences from CPV-2a were the substitution of two amino acid $(\mathrm{Asn} \rightarrow \mathrm{Asp}, \mathrm{Ile} \rightarrow \mathrm{Val})$ in the VP2 protein. Both of these biotypes are now prevailing throughout the canine population worldwide, replacing the original strain, CPV-2 [7, 13, 18]. Nevertheless, epidemiological study of CPV-2a and CPV-2b in Japan has not been well investigated.

In this study, we isolated CPV-2b from a diarrheic fecal sample from a puppy and investigated the phylogenetic relation between the clinical isolate and the other CPVs by molecular analysis of the VP2 capsid gene.

A 5-month-old male Miniature Dachshund weighing 3.5 $\mathrm{kg}$ was referred to Nihon University Animal Medical Center with complaints of depression, fever, anorexia, diarrhea and vomiting. Complete blood cell count revealed leukopenia $(4700$ cells $/ \mu l)$ and the results of a serum biochemical analysis were within the normal range (Table 1). A parvoviral infection was confirmed by a fecal examination with a parvoviral antigen detection kit (Sinovus Biotech Inc., N.Y., U.S.A.).

The patient dog was treated with enrofloxacin $(5 \mathrm{mg} / \mathrm{kg})$ for 3 days. After a 7-day treatment, the dog recovered from

\footnotetext{
* Correspondence to: Dr. Kano, R., Department of Pathobiology, Nihon University School of Veterinary Medicine, 1866, Kameino, Fujisawa Kanagawa, 252-8510, Japan.
}

vomiting and had a good appetite.

The fecal sample was collected from the patient dog and suspended in phosphate-buffered saline (PBS). After centrifugation at $900 \mathrm{G}$ for $20 \mathrm{~min}$, the supernatant fluid was collected. This supernatant was filtered with a $0.45-\mu \mathrm{m}$ filter (Millipore Co., Mass, U.S.A.). The filtered sample was overlaid on a Crandell feline kidney (CRFK) cell line grown in Dulbecco's modified Eagle's medium (DMEM) supplemented with $10 \%$ fetal bovine serum (FBS) and antibiotics (at final concentrations of 100 units $/ \mathrm{m} l$ of penicillin G potassium and $0.5 \mathrm{mg} / \mathrm{m} l$ of streptomycin sulfate). This CRFK had been seeded about $24 \mathrm{hr}$ earlier at a density of 4 $\times 10^{4}$ cells $/ \mathrm{m} l$ before overlaying. After several days, a cytopathic effect (CPE) in the cells was observed. The cells were frozen and thawed three times. The cell debris was removed by centrifugation at $900 \mathrm{G}$ for $20 \mathrm{~min}$. The supernatant fluid was filtered with a $0.45-\mu \mathrm{m}$ filter and the filtered sample was collected. The virus in the collected sample was passaged several times in the CRFK cells before examination.

Antigenic analysis was performed by hemagglutinating (HA) test and hemagglutination-inhibition (HI) test according to Carmichael et al. [4].

The CPV VP2 gene specific primer pairs were used in this study as reported by Ikeda et al. [9]. The sequences of the primers were as follows:

$$
\begin{aligned}
& 51 \text { (5'-CCAACTAAAAGAAGTAAACC-3') } \\
& \text { F1 (5'-AGATAGTAATAATACTATGCCATTT-3') } \\
& \text { F2 (5'-ACAGGAGAAACACCTGAGAGATTTA-3') } \\
& \text { R1 (5'-TGGTTGGTTTCCATGGATAAAAACC-3') } \\
& \text { R2 (5'-TTTTGAATCCAATCTCCTTCTGGAT-3') } \\
& \text { R3 (5'-CCTATATCAAATACAAGTACAATA-3') }
\end{aligned}
$$

To amplify the VP2 gene of CPV by PCR, the following primer pairs were used: primer pair A [primers 51 and R3] for amplifying the regions between nt 2687 and 4692, primer pair B [primers 51 and R1] for amplifying the regions between nt 2687 and 3395, primer pair C [primers F1 and R2] for amplifying the regions between nt 3316 and 4035 , and primer pair D [primers F2 and R3] for amplifying 
the regions between nt 3956 and 4692 .

Samples which contained the virus as a template were directly added to the PCR mixture without the extraction of the virus DNA. The PCR reaction mixture $(30 \mu l)$ contained $10 \mathrm{mM}$ Tris- $\mathrm{HCl}(\mathrm{pH} 8.3), 50 \mathrm{mM} \mathrm{KCl}, 1.5 \mathrm{mM} \mathrm{MgCl}_{2}$, $0.001 \%$ gelatin and $2.5 \mathrm{mM}$ each of deoxynucleoside triphosphate, 1.0 unit of Taq polymerase and $0.5 \mu \mathrm{g}$ of a pair of primers. The PCR amplification was carried out for 30 cycles and consisted of template denaturation $(0.5 \mathrm{~min}$, at $\left.94^{\circ} \mathrm{C}\right)$, primer annealing $\left(2 \mathrm{~min}\right.$, at $\left.55^{\circ} \mathrm{C}\right)$ and polymerization $\left(2 \mathrm{~min}\right.$, at $\left.74^{\circ} \mathrm{C}\right)$. The PCR products were electrophoresed through $2 \%$ agarose gel and then stained with ethidium bromide. The PCR products from the samples were sequenced by the dideoxy chain termination method with an ABI PRISM 310 Genetic Analyzer (Perkin-Elmer, Foster City, CA, U.S.A.).

Homology relationships among the VP2 gene of reference strains of CPV were examined by FASTA database analysis in the DNA data bank of Japan (DDBJ).

To examine the VP2 gene among clinical isolates and the other CPVs, we performed the neighbor-joining (NJ) method in the Clustal $\mathrm{W}$ multiple sequence alignment programs [17]. The phylogenic tree was constructed by meams of the TREEVIEW program [12]. Bootstrap analysis was performed on 1000 random samples taken from multiple alignment as described by Felsenstein [6], and analyzed by the Clustal W programs.

The results of serological analyses (HA test and HI test) of the clinical isolate are shown in Table 2.

Amplification of isolate of CPV VP2 gene with 6 primers yielded 1755-bp fragments (Fig. 1), consistent with the expected sizes of the CPV VP2 gene reported previously [9].
Table 1. Results of complete blood cell count and serum biochemical analysis

\begin{tabular}{llrllc}
\hline ALB & $\mathrm{g} / \mathrm{d} l$ & 2.3 & WBC & $/ \mu l$ & 4700 \\
\hline ALKP & $\mathrm{U} / \mathrm{L}$ & 191 & RBC & $\times 10^{4} / \mu l$ & 621 \\
\hline ALT & $\mathrm{U} / \mathrm{L}$ & 55 & HGB & $\mathrm{g} / \mathrm{d} l$ & 14.2 \\
\hline AMYL & $\mathrm{U} / \mathrm{L}$ & 11010 & HCT & $\%$ & 39.7 \\
\hline BUN & $\mathrm{mg} / \mathrm{d} l$ & 11.3 & MCV & $\mathrm{fl}$ & 63.9 \\
\hline $\mathrm{Ca}$ & $\mathrm{mg} / \mathrm{d} l$ & 9.4 & $\mathrm{MCH}$ & $\mathrm{pg}$ & 22.9 \\
\hline CHOL & $\mathrm{mg} / \mathrm{d} l$ & 186.9 & $\mathrm{MCHC}$ & $\mathrm{g} / \mathrm{d} l$ & 35.8 \\
\hline CREA & $\mathrm{mg} / \mathrm{d} l$ & 0.6 & $\mathrm{PLT}$ & $\times 10^{4} / \mu l$ & 38 \\
\hline GLU & $\mathrm{mg} / \mathrm{d} l$ & 121.7 & $\mathrm{CRP}$ & $\mathrm{mg} / \mathrm{d} l$ & 0.25 \\
\hline LIPA & $\mathrm{U} / \mathrm{L}$ & 2910 & & & \\
\hline $\mathrm{NH} 3$ & $\mathrm{umol} / \mathrm{L}$ & 25 & $\mathrm{Na}$ & $\mathrm{mmol} / \mathrm{L}$ & 139 \\
\hline TP & $\mathrm{g} / \mathrm{d} l$ & 5.1 & $\mathrm{~K}$ & $\mathrm{mmol} / \mathrm{L}$ & 4.9 \\
\hline GLOB & $\mathrm{g} / \mathrm{d} l$ & 2.7 & $\mathrm{Cl}$ & $\mathrm{meq} / \mathrm{L}$ & 101 \\
\hline
\end{tabular}

Table 2. Comparison of CPV hemagglutination (HA) and hemagglutination-Inhibition (HI) titers

\begin{tabular}{lccc}
\hline \multirow{2}{*}{ Test virus } & \multicolumn{2}{c}{ at first admission } & after 1 week \\
& HA & HI & HI \\
\hline CP49 & 1280 & 512 & 256 \\
TDK & 1280 & 512 & 256 \\
Y-1 & 640 & 512 & 256 \\
Clinical Isolate & 160 & 1024 & 256 \\
\hline
\end{tabular}

CP49 (strain of CPV-2), TDK (strain of CPV-2a), Y-1 (strain of $\mathrm{CPV}-2 \mathrm{~b}$ ), Clinical Isolate (strain of CPV-2b).

Table 3. Phylogenetically informative nucleotide sequence of VP2 gene. The coding sequence differences in the VP2 protein gene which were found in the various isolates

\begin{tabular}{lcccccccccccccccccc}
\hline $\begin{array}{l}\text { nucleotide } \\
\text { Biotype }\end{array}$ & 239 & 246 & 259 & 279 & 308 & 699 & 871 & 889 & 899 & 913 & 967 & 1038 & 1167 & 1276 & 1623 & 1691 & 1703 \\
\hline FPLV & & A & A & A & A & T & T & T & C & C & G & G & A & T & A & A & A & C \\
CPV-2 & G & G & A & C & C & C & C & C & C & G & A & G & C & A & A & G & G \\
CPV-2a & G & G & T & C & C & C & C & G & G & T & A & G & C & A & C & G & G \\
CPV-2b & G & G & T & C & C & C & C & G & G & T & A & G & C & G & C & G & G \\
CPV-2c & G & G & T & C & C & C & C & G & A & T & A & G & C & A $\backslash$ G & C & G & G \\
Clinical Isolate & G & G & T & C & C & C & C & G & G & T & A & G & C & G & C & G & G \\
\hline
\end{tabular}

Table 4. Phylogenetically informative amino acid sequence of VP2 gene

\begin{tabular}{lcccccccccccccc}
\hline $\begin{array}{l}\text { Amino acid } \\
\text { Biotype }\end{array}$ & 80 & 87 & 93 & 103 & 232 & 297 & 300 & 305 & 323 & 426 & 555 & 564 & 568 \\
\hline FPLV & Lys & Met & Lys & Val & Val & Ser & Ala & Asp & Asp & Asn & Val & Asn & Ala \\
CPV-2 & Arg & Met & Asn & Ala & Ile & Ser & Ala & Asp & Asn & Asn & Val & Ser & Gly \\
CPV-2a & Arg & Leu & Asn & Ala & Ile & Ser/Ala & Gly & Tyr & Asn & Asn & Ile & Ser & Gly \\
CPV-2b & Arg & Leu & Asn & Ala & Ile & Ser/Ala & Gly & Tyr & Asn & Asp & Val & Ser & Gly \\
CPV-2c & Arg & Leu & Asn & Ala & Ile & Ala & Asp & Tyr & Asn & Asn/Asp & Val & Ser & Gly \\
Clinical Isolate & Arg & Leu & Asn & Ala & Ile & Ala & Gly & Tyr & Asn & Asp & Val & Ser & Gly \\
\hline
\end{tabular}

FPLV=Feline panleukopenia virus; $\mathrm{CPV}=$ Canine parvovirus. 
ATGAGTGATGGAGCAGTTCAACCAGACGGTGGTCA $\$$ CTGCTGTCAGAAATGAAAGAGCT

$\begin{array}{llllllllllllllllllll}M & S & D & G & A & V & \& & \text { P } & D & G & G & Q & \text { P } & A & \text { V } & R & \text { N } & \text { E } & R & A\end{array}$ ACAGGATCTGGGAACGGGTCTGGAGGCGGGGGTGGTGGTGGTTCTGGGGTGTGGGGATT

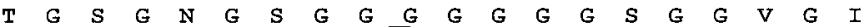
TCTACGGTACTTTCAATAATCAGACGAAATTTAAATTITTGGAAAACGGATGGGTGGAA $\begin{array}{lllllllllllllllllllll}S & T & G & T & F & N & N & Q & T & E & F & K & F & \text { L } & E & \text { N } & G & \text { W } & \text { V } & \text { E }\end{array}$ ATCACAGCAAACTCAAGCAGACTIGTACATTTAAATATGCCAGAAAGTGAAAATTATAGA $\begin{array}{lllllllllllllllllllll}I & T & A & N & S & S & R & L & V & H & L & N & M & P & E & S & E & N & Y & R\end{array}$ AGAGTGGTTGTAAATAATTTGGATAAAACTGCAGTTAACGGAAACATGGCTTTAGATGAT $\begin{array}{lllllllllllllllllllll}R & V & V & V & N & N & L & D & K & T & A & V & N & G & N & M & A & I & D & D\end{array}$ ACCCATGCACAAATTGTAACACCTTGGTCATTGGTTGATGCAAATGCTTGGGGAGTTTGG

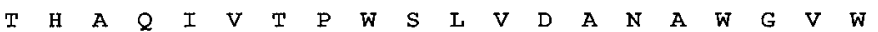
TTTAATCCAGGAGATTGGCAACTAATTGTTAATACTATGAGTGAGTTGCATTTAGTTAGT $\begin{array}{lllllllllllllllllllll}F & N & P & G & D & W & \& & L & I & V & N & T & M & S & E & L & H & I & V & S\end{array}$ TITGAACAAGAAATTTTTAATGTTGTTTTIAAAGACTGTTTCAGAATCTGCTACTCAGCCA $\begin{array}{llllllllllllllllllllll}F & E & Q & E & I & F & N & V & V & L & K & T & V & S & E & S & A & T & Q & P\end{array}$ CCAACTAAAGTTTATAATAATGATTTAACTGCATCATTGATGGTTGCATTAGATAGTAAT

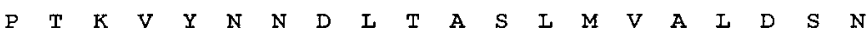
AATACTATGCCATTTACTCCAGCAGCTATGAGATCTGAGACATTGGGTTTTTATCCATGG $\begin{array}{lllllllllllllllllllll}N & T & M & P & F & \text { T } & \text { P } & \text { A } & \text { A } & \text { M } & \text { R } & \text { S } & \text { E } & \text { T } & \text { L } & \text { G } & \text { F } & \text { Y } & \text { P } & \text { W }\end{array}$ AAACCAACCATACCAACTCCATGGAGATATTATTTTTCAATGGGATAGAACATTAATACCA $\begin{array}{lllllllllllllllllllllll}K & P & T & I & P & T & P & W & R & Y & Y & F & Q & W & D & R & T & L & I & P\end{array}$ TCTCATACTGGAACTAGTGGCACACCAACAAATATATACCATGGTACAGATCCAGATGAT $\begin{array}{lllllllllllllllllllll}S & \text { H } & \text { T } & G & \text { T } & S & G & T & P & \text { T } & \text { N } & \text { I } & Y & \text { H } & G & \text { T } & \text { D } & P & \text { D } & D\end{array}$ GTTCAATTTTATACTATTGAAAATTCTGTGCCAGTACACTTACTAAGAACAGGTGATGAA $\begin{array}{lllllllllllllllllllllll}V & Q & F & Y & T & I & E & N & S & V & P & V & H & L & L & R & T & G & D & E\end{array}$ TITGCTACAGGAACATTTTTTTTTGATIGTAAACCATGTAGACTAACACATACATGGCAA $\begin{array}{llllllllllllllllllllll}F & A & T & G & T & F & F & F & D & C & K & P & C & R & L & T & H & T & W & Q\end{array}$ ACAAATAGAGCATTGGGCTTACCACCATTTCTAAATTCTTTTGCCTCAAGCTGAAGGAGGT

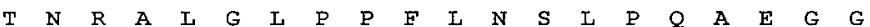
ACTAACTTTGGTTATATAGGAGTTCAACAAGATAAAAGACGTGGTGTAACTCAAATGGGA

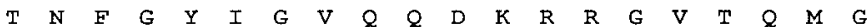
AATACAAAT TATATTACTGAAGCTACTATTATGAGACCAGCTGAGGTTGGTATAGTGCA $\begin{array}{llllllllllllllllllll}N & T & \mathbb{N} & Y & I & T & E & A & T & I & M & R & P & A & E & V & G & Y & S & A\end{array}$ CCATATTATTCTTTTGAGGCGTCTACACAAGGGCCATTTAAAACACCTATTGCAGCAGGA $\begin{array}{lllllllllllllllllllll}P & Y & Y & S & F & E & A & S & T & Q & G & P & F & K & T & P & I & A & A & G\end{array}$ CGGGGGGGAGCGCAAACAGATGAAAATCAAGCAGCAGATGGTGATCCAAGATATGCATTT $\begin{array}{llllllllllllllllllll}R & G & G & A & Q & T & D & E & N & Q & A & A & D & G & D & P & R & Y & A & F\end{array}$ GGTAGACAACATGGTCAAAAAACTACCACAACAGGAGAAACACCTGAGAGATTTACATAT $\begin{array}{lllllllllllllllllllll}G & R & \odot & H & G & O & K & T & T & T & T & G & E & T & P & E & R & F & T & Y\end{array}$ ATAGCACATCAAGATACAGGAAGATATCCAGAAGGAGATTGGATTCAAAATATTAACTTT

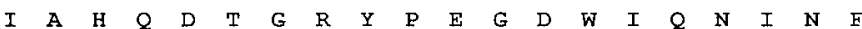
AACCTTCCTGTAACAGATGATAATGTATT TIACCAACAGATCCAATTGGAGGTAAAACA $\begin{array}{llllllllllllllllllllll}N & L & P & V & T & D & D & N & V & L & L & P & T & D & P & I & G & G & K & T\end{array}$ GGAATTAACTATACTAATATATTTAATACTTATGGTCCTTTAACTGCATTAAATAATGTA $\begin{array}{lllllllllllllllllllll}G & I & N & Y & T & N & I & F & N & T & Y & G & P & L & T & A & L & N & N & V\end{array}$ CCACCAGTTTATCCAAATGGTCAAATTTGGGATAAAGAATTTGATACTGACTTAAAACCA

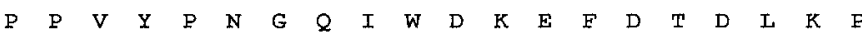
AGACTTCATGTAAATGCACCATTTGTTTGTCAAAATAATTGTCCTGGTCAATTATTTGTA $\begin{array}{lllllllllllllllllllll}R & L & H & V & N & A & P & F & V & C & Q & N & N & C & P & G & Q & L & F & V\end{array}$ AAAGTTGqAFCTAATTIAACAAATGAATATGATCCIGATGCATCTGCTAATATGTCAAGA $\begin{array}{llllllllllllllllllll}K & V & A & P & \text { N } & \text { L } & \text { T } & \text { N } & \text { E } & \text { Y } & \text { D } & \text { P } & \text { D } & \text { A } & S & \text { A } & \text { N } & \text { M } & \text { S } & R\end{array}$ ATTGTAACTTACTCAGATTITTGGTGGAAAGTAAATTAGTATTTAAAGCTAAACTAAGA 1620 $\begin{array}{llllllllllllllllllllllllllll}I & V & T & Y & S & D & F & W & W & K & G & K & L & V & F & K & A & K & L & R\end{array}$ GCCTCTCATACTTGGAATCCAATTCAACAAATGAGTATTAATGTAGATAACCAATTTAAC 1680 $\begin{array}{lllllllllllllllllllllllllllll}A & S & H & T & W & N & P & I & Q & Q & M & S & I & N & V & D & N & Q & F & N\end{array}$ TATGTACCAAGTAAE TTtGGAGGTATGAAAATTGTATATGAAAAATCTCAACTAGCACCT $\begin{array}{lllllllllllllllllllll}Y & V & P & S & N & I & G & G & M & K & I & V & Y & E & K & S & Q & L & A & P\end{array}$ AGAAAATTATATTAAA

Fig. 1. Nucleotide sequence of the VP2 gene of the clinical isolate in this study. The boxed sequence indicates the specific differences between the nucleotide of a clinical isolate and another CPV-2b obtained from the GeneBank database. 


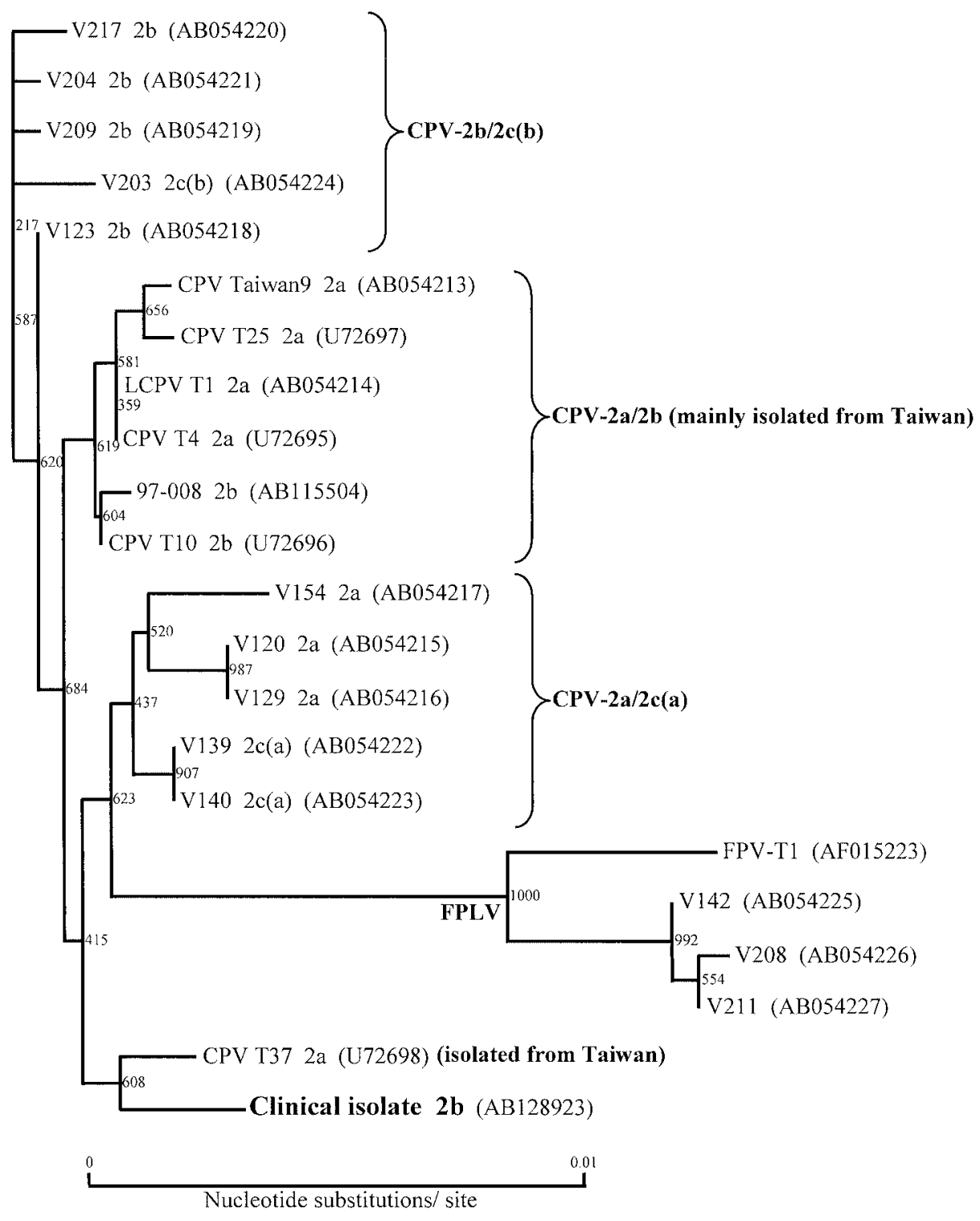

Fig. 2. Phylogenetic tree constructed from the VP2 gene nucleotide sequences of a clinical isolate in this study and other sequences obtained from the GenBank datebase.

The nucleotide differences in the VP2 gene between the clinical isolate in this study [DDBJ accession no. AB128923 (Fig. 1)] and common strains of CPV-2b were detected in 6 bases [nucleotide $36(\mathrm{~A} \rightarrow \mathrm{G})$, nucleotide $147(\mathrm{~A} \rightarrow \mathrm{G})$, nucleotide $969(\mathrm{C} \rightarrow \mathrm{T})$, nucleotide $1291(\mathrm{C} \rightarrow \mathrm{T})$, nucleotide $1509(\mathrm{G} \rightarrow \mathrm{A})$ and nucleotide $1695(\mathrm{~T} \rightarrow \mathrm{C})]$. The relation among antigenic typings of CPV and the specific nucleotide of VP2 protein is shown in Tables 3 and 4 . These analyses confirmed that the isolated CPV was CPV-2b in the antigenic type.

The phylogenetic analysis of CPV for VP2 gene sequences of the clinical isolate and the others revealed that the clinical isolate is genetically close to the wild-type CPV- 2a isolate from Taiwan (Fig. 2).

The puppy in this case had been vaccinated twice, but the patient was infected with CPV, showing the symptoms. The antigen type of the isolate was CPV-2b, which has become common throughout the world, including Japan, in recent years. The vaccination program has been designed to protect dogs from CPV-2b infection, but the commercial vaccines sometimes failed, as in this case. Therefore, it is speculated that there might be some differences in the antigen epitope among field isolates of CPV.

The homology of VP2 gene nucleotide sequences was $99.6 \%$ between the clinical isolate and 3 reference strains [CPV-T37 (dog, Taiwan), CPV-T10 (dog, Taiwan), V123 
(cat, Vietnam)], and was $99.5 \%$ between the clinical isolate and strain 97-008 (dog, Japan) isolated from Japanese domestic dogs in recent years. Moreover, the phylogenetic analysis of the VP2 gene indicated that this clinical isolate was close to the isolate of wild-type CPV from Taiwan (strain CPV T-37) rather than the isolates from Japan. This CPV-2b isolate might have been imported from Taiwan, since no isolates like the strains prevailing in Taiwan have been detected in Japan.

\section{REFERENCES}

1. Azetaka, M., Hirasawa, T., Konishi, S. and Ogata, M. 1981. Jpn. J. Vet. Sci. 43: 243-255.

2. Battilani, M., Ciulli, S., Tisato, E. and Prosperi, S. 2002. Virus Res. 83: 149-157.

3. Battilani, M., Scagliarini, A., Tisato, E., Turilli, C., Jacoboni, I., Casadio, R. and Prosperi, S. 2001. J. Gen. Virol. 82: 15551560.

4. Carmichael, L. E., Joubert, J. C. and Pollock, R. V. H. 1980. Am. J. Vet. Res. 41: 784-791.

5. Chalmers, W. S. K., Truyen, U., Greenwood, N. M. and Baxendale, W. 1999. Vet. Microbiol. 69: 41-45.

6. Felsenstein, J. 1985. Evolution 39: 783-791.

7. Ikeda, Y., Nakamura, K., Miyazawa, T., Tohya, Y., Takahashi,
E. and Mochizuki, M. 2002. Emerg. Infect. Dis. 8: 341-346.

8. Ikeda, Y., Miyazawa, T., Kurosawa, K., Naito, R., Hatama, S., Kai, C. and Mikami, T. 1998. J. Vet. Med. Sci. 60: 973-974.

9. Ikeda, Y., Mochizuki, M., Naito, R., Nakamura, K., Miyazawa, T., Mikami, T. and Takahashi, E. 2000. Virology 278: 13-19.

10. Mochizuki, M., Horiuchi, M., Hiragi, H., SanGabriel, MC., Yasuda, N. and Uno, T. 1996. J. Clin. Microbiol. 34: 21012105.

11. Nakamura, K., Sakamoto, M., Ikeda, Y., Sato, E., Kawakami, K., Miyazawa, T., Tohya, Y., Takahashi, E., Mikami, T. and Mochizuki, M. 2001. Clin. Diagn. Lab. Immunol. 8: 663-668.

12. Page, R. D. 1996. Comput. Appl. Biosci. 12: 357-358.

13. Parker, J. S. L. and Parrish, C. R. 1997. J. Virol. 71: 9214 9222.

14. Pratelli, A., Cavalli, A., Martella, V., Tempesta, M., Decaro, N., Carmichael, L. E. and Buonavoglia, C. 2001. Clin. Diagn. Lab. Immunol. 8: 612-615.

15. Rypula, K., Chmielewski, R., Smielewska-Los, E. and Klimentowski, S. 2002. J. Vet. Med. B. Infect. Dis. Vet. Public. Health. 49: $142-145$.

16. Steinel, A., Munson, L., Van Vuuren, M. and Truyen, U. 2000. J. Gen. Virol. 81: 345-350.

17. Thompson, J. D., Higgins, D. G. and Gibson, T. J. 1994. Nuc. Acid. Res. 22: 4673-4680.

18. Truyen, U. 1999. Vet. Microbiol. 69: 47-50. 